This item was submitted to Loughborough's Research Repository by the author.

Items in Figshare are protected by copyright, with all rights reserved, unless otherwise indicated.

\title{
A hybrid patient-specific biomechanical model based image registration method for the motion estimation of lungs
}

PLEASE CITE THE PUBLISHED VERSION

https://doi.org/10.1016/j.media.2017.04.003

\section{PUBLISHER}

(C) Elsevier

VERSION

AM (Accepted Manuscript)

\section{PUBLISHER STATEMENT}

This work is made available according to the conditions of the Creative Commons Attribution-NonCommercialNoDerivatives 4.0 International (CC BY-NC-ND 4.0) licence. Full details of this licence are available at: https://creativecommons.org/licenses/by-nc-nd/4.0/

\section{LICENCE}

CC BY-NC-ND 4.0

\section{REPOSITORY RECORD}

Han, Lianghao, Hua Dong, Jamie R. McClelland, Liangxiu Han, David J. Hawkes, and Dean C. Barratt. 2019. "A Hybrid Patient-specific Biomechanical Model Based Image Registration Method for the Motion Estimation of Lungs". figshare. https://hdl.handle.net/2134/35031. 
1 A Hybrid Patient-Specific Biomechanical Model Based Image Registration Method for the Motion Estimation of Lungs

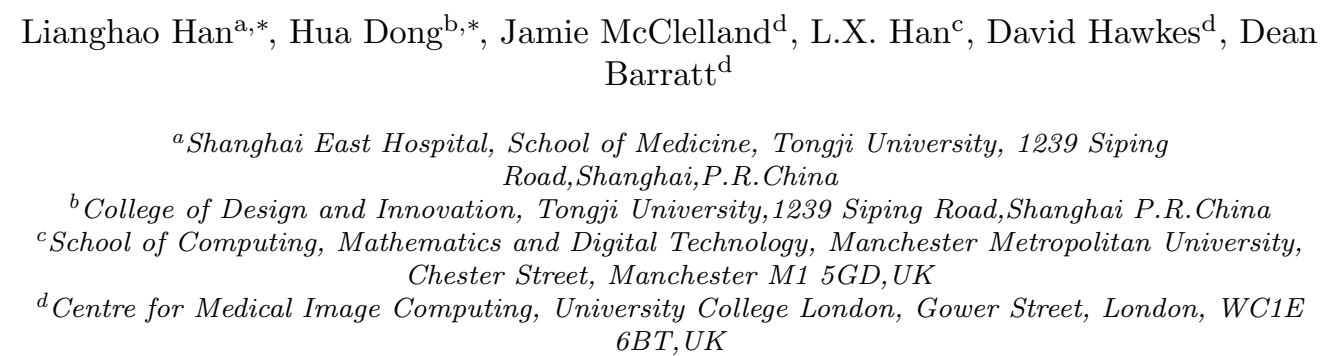

\section{${ }_{12}$ Abstract}

This paper presents a new hybrid biomechanial model-based non-rigid image registration method for lung motion estimation. In the proposed method, a patient-specific biomechanical modelling process captures major physically realistic deformations with explicit physical modelling of sliding motion, whilst a subsequent non-rigid image registration process compensates for small residuals. The proposed algorithm was evaluated with 10 4D CT datasets of lung cancer patients. The target registration error (TRE), defined as the Euclidean distance of landmark pairs, was significantly lower with the proposed method $(\mathrm{TRE}=1.37 \mathrm{~mm})$ than with biomechanical model $(\mathrm{TRE}=3.81 \mathrm{~mm})$ and intensity-based image registration without specific considerations for sliding motion $(\mathrm{TRE}=4.57 \mathrm{~mm})$. The proposed method achieved a comparable accuracy as several recently developed intensity-based registration algorithms with sliding handling on the same datasets. A detailed comparison on the distributions of TREs with three non-rigid intensity-based algorithms showed that the proposed method performed especially well on estimating the displacement field of lung surface regions (mean TRE $=1.33 \mathrm{~mm}$, maximum TRE $=5.3 \mathrm{~mm}$ ). The effects of biomechanical model parameters (such as Poisson's ratio, friction and tissue heterogeneity) on displacement estimation were investigated. The potential of the algorithm in optimising biomechanical models of lungs through analysing the pattern of displacement compensation from the image registration process has also been demonstrated. 

registration, $4 \mathrm{D}$ CT, Lung

\section{Introduction}

Respiratory motion can cause artefacts in images during thorax and abdomen imag17 ing. Accurate estimation and correction for the effects of respiratory motion can potentially increase the applications of medical images in diagnosis, treatment planning and

* Corresponding author

Email addresses: 1hhan@tongji.edu.cn (Lianghao Han), donghua@tongji.edu.cn (Hua Dong), 1.han@mmu.ac.uk (L.X. Han), d.hawkes@ucl.ac.uk (David Hawkes), d.barratt@ucl.ac.uk> (Dean Barratt) 
and overlaps between nearby voxels may appear near the sliding interfaces. To reduce

39 or eliminate gaps between the independently registered regions, a boundary-matching

40 penalty method has been proposed in which an artificial uniform band with a unique

${ }_{41}$ intensity value around the sub-regions is added (McClelland et al. 2006; Wu et al.

42 2008, Vandemeulebroucke et al. 2012). This has the effect of creating a strong spa-

43 tial gradient around the sliding interface, which guides each registration, resulting in

${ }_{44}$ greater consistency at the interfaces. An alternative approach is to incorporate a spe-

45 cific regularisation of sliding motion into the registration optimisation. Such schemes

46 are mainly based on the consideration that the deformation components in the nor-

47 mal and tangential directions near to sliding boundaries have different contributions to

48 sliding motion; the sliding behaviour is mainly controlled by the tangential component.

49 For example, Schemit-Richberg et al. (Schmidt-Richberg et al. 2012a|b) described a

${ }_{50}$ direction-dependent diffusion regularisation approach. In their method, the tangential

51 component was smoothed separately for the two adjacent regions on either side of their

52 common sliding interfaces, whilst the normal component was smoothed jointly across

53 the two regions. This allows a discontinuous movement between the two sub-regions in

${ }_{54}$ the tangential direction but maintains smoothness in the normal direction to reduce gaps

55 and overlaps. Similarly, Delmon et al. (Delmon et al. 2013) proposed a B-spline registra-

56 tion method with direction-dependent B-spline decomposition for sliding regularisation.

57 They used a B-spline transformation for each sub-region to capture the discontinuities

58 of displacement due to sliding motion between two sub-regions. Pace et al. (Pace et al.,

59 2011) presented an anisotropic diffusive regularisation method in which separate nonlin-

${ }_{60}$ ear anisotropic smoothing filters were applied to the normal and tangential deformation

61 components of displacement. More recently, Riser et al. (Risser et al. 2013) proposed a

62 direction-dependent regularisation within a diffeomorphic registration framework, similar

63 to Schemit-Richberts methods. However, they decomposed the velocity field rather than

64 the displacement field into normal and tangential components. To consider discontinu-

65 ities of deformation existing in both normal and tangent directions around boundaries

${ }_{66}$ between lung lobe fissures, Yin et al. (Yin et al. 2010) used a diffusive regularisation with

67 an additional distance weighting term increasing with the distance to the organ bound-

68 ary. This ensures that the displacement discontinuity characteristics of sliding motion 
near organ boundaries are not penalized.

All the above methods require segmenting sliding structures in the images to be registered. To address this pre-requisite, Schemit-Richberg et al. (Schmidt-Richberg et al. 2012b) extended their method, demonstrating that it was possible to automatically detect sliding organs, thus removing the requirement for prior image segmentation which may be impractical or overly time-consuming for some clinical applications. Several intensity-based image regularisation methods that do not require a prior segmentation have also been proposed to preserve sliding motion. Based on the decomposition of the displacement vector, Ruan et al. (Ruan et al. 2009), for instance, proposed a regularisation energy function written as a combination of an L2 norm of the divergence of the displacement vector (i.e. the relative variation of the volume) and an L1 norm of a rotational vector (i.e. the curl of the displacement field). Penalizing the L2 norm conserves the volume change, whilst penalizing the L1 norm preserves large shear along the boundaries. Further, Heinrich et al. (Heinrich et al. 2010) showed that a nonquadratic regularisation using the $\operatorname{L} p$ norm $(p<=1)$ can preserve the sliding motion of lungs within an optical flow based registration algorithm. More recently, Heinrich et al. (Heinrich et al., 2013) introduced an intensity-derived minimum-spanning tree into their Markov random field (MRF) based deformable registration method to represent the underlying structure of the anatomical connectivity of the image. A pair-wise regularisation acts only on connections (edges) between two nodes of the tree. Using this method, the sliding motion is preserved. However, since sliding motion is not handled explicitly, all of these methods require large variations of image gradient at the sliding boundaries in order to preserve the sliding motion. Therefore, such regularisation approaches may be insufficient when the image intensities are similar near the interface between two sliding objects; for example, at the boundary between the chest wall and the liver in CT or MR images.

\subsection{Biomechanical model based methods for lung motion estimation}

Biomechanical modelling is another commonly used approach for estimating lung motion. In the biomechanical modelling techniques, the sliding motion between two anatomic structures is often treated as a frictional or frictionless contact problem, which then is solved using finite element (FE) methods. Zhang et al. (Zhang et al., 2004) 
proposed a deformable lung FE model with pleural sliding using contact elements in the commercial FE package ANSYS (http://www.ansys.com). Lung expansion from the end exhalation to the end inhalation was simulated by applying a negative uniform pressure to the external lung surface until it fills the chest cavity; the interaction between the lungs and their surrounding body was modelled explicitly as a contact problem. The feasibility of this approach was demonstrated by using two 3D breathhold lung CT images, acquired from one patient at the exhalation phase and at the deep inspiration phase. Villard et al. (Villard et al., 2005) described a similar FE model for deformable lung registration. Pleural sliding between the rib cage and lungs was modelled as a frictionless contact using the open source FE software toolkit, Code_Aster (www.code-aster.org). Following the ideas of Zhang and Villard on lung FE models, Werner et al. (Werner et al., 2009a) simulated the lung expansion under a negative pressure using the commercial FE software package, COMOSOL Multiphysics (http://www.comsol.ltd.uk), and provided a detailed quantitative evaluation of their lung model using CT datasets from 12 lung tumour patients. The results suggested that an FE modelling approach was adequate in predicting lung dynamics due to lung ventilation, even lung tissue was assumed to be an isotropic, homogeneous and linearly elastic material. More recently, Fuerst et al. (Fuerst) et al. 2015) simulated the lung expansion from the end-exhale to the end-inspiration by applying different negative pressures on the pre-defined surface zones of thorax and diaphragm contacting with lungs, respectively. The applied pressures were then transformed to the lung surface through a lung/thorax/diaphragm interaction model, whose values were estimated through an optimisation procedure where the model-estimated lung change was compared to CT images at end-inspiration. The sliding between the lung and the surfaces of thorax cavity and diaphragm was simulated as a frictionless contact problem.

Biomechanial modelling of lung respiration has also been treated as a compression process from inspiration to expiration. Using a frictionless contact model, Al-Mayah et al. (Al-Mayah et al., 2008) modelled the lung sliding against the chest cavity using the commercial FE software package, ABAQUS (http://www.3ds.com). In this case, they simulated lung respiration as a compression process from inspiration to expiration by applying displacement boundary conditions to the inner surface of the chest cavity directly 
in contact with the lung surface. They used the commercial mesh manipulation tool, Hypermorph (http://www.altairhyperworks.com), to deform the surface mesh of the chest cavity at end inhalation to match the surface mesh at end-exhalation, and obtain the displacements of each node on the surface mesh of the chest cavity at end inhalation. These nodal displacements were then used as displacement boundary conditions to deform the lungs in the FE models. Using similar FE models, they investigated the effects of friction near the interface (Al-Mayah et al. 2009), the heterogeneity of lung structures (Al-Mayah et al. 2010), linear/nonlinear material models (Al-Mayah et al. 2008 ) and material parameters of lung tissues (Al-Mayah et al. 2009).

Compared to intensity-based image registration techniques, biomechanical modelling often has lower requirements for image quality and can work on noisy images, such as ultrasound, since the generation of biomechanical models in many applications only requires organ surface data from images. Another attractive feature is that biomechanical modelling can provide an integrated solution in one single model for physics and physiology based lung motion, including but not limited to, predicting the deformation/motion of tumours, evaluating the effect of gravity on respiratory physiology, simulating biophysiological processes, such as respiratory motion, and providing physically realistic sliding motion, including explicitly information on physical properties and mechanical behaviour of anatomical structures, with or without pathology, if they are available. Under the framework of non-rigid intensity-based image registration, usually each specific regularization technique has to be developed for each individual physical or physiologic property in order to provide physically realistic deformation estimations, and developing an integrated solution for various properties is a challenging task. However, due to various uncertainties, such as forces exerted by the beating heart, variable lung and blood pressure, and variable mechanical properties of in vivo tissues, which are in general very difficult to measure accurately, combined with limited tissue contrast in some image modalities and limited computational time and resources, a number of simplifications and assumptions are required when generating biomechanical models. Unlike imageintensity based image registration methods, it is also extremely difficult, if not impossible, for biomechanical models to include very detailed internal tissue structures whose deformations may directly manifest as intensity changes in medical images. All of these 
factors limit the accuracy of biomechanical models in predicting displacement distributions of tissue structures. Previous lung motion studies show that biomechanical models only achieve equivalent prediction accuracy as intensity-based image registration methods without sliding motion regularisation, but exhibit inferior registration performance compared with intensity-based image registration methods with sliding regularisation in terms of landmark-based TRE (Werner et al., 2009a b). Therefore, intensity-based image registration methods and biomechanical modelling have their own advantages and disadvantages when applied to lung motion estimation involving interface sliding, but an important observation that underlies the work described in the present paper is that the advantages of these methods are potentially complementary.

Previous studies (Li et al., 2008; Han et al., 2014b, Samavati et al. 2015, Hipwell et al. 2016) have shown that a combined method integrating intensity-based registration with biomechanical modelling can compensate physically unrealistic estimated tissue motion (Li et al. 2008), reduce the uncertainty of biomechanical modelling ((Samavati et al. 2015) ), compensate displacement residuals ((Han et al., 2014b) ) due to the simplification of biomechanical models, and improve the registration performance by increasing image overlap (Han et al., 2014b: Hipwell et al., 2016). Our recent preliminary studies on deformable registration of CT lung images have demonstrated a good registration performance using a combined method (Han et al. 2014a), in which an intensity-based image registration process provides a displacement compensation to displacement residues of biomechanical modelling. Since the displacement compensation reflects the distributions of the prediction errors of biomechanical modelling, which potentially could be used to provide directions for optimising model parameters and constructing more accurate predictive biomechanical models.

In the present study, we propose a patient-specific, hybrid biomechanical modelbased image registration method for lung motion estimation, as an extension of the work reported in (Han et al., 2014a). In this method, a biomechanical modelling process with an FE method estimates the major component of the deformation field from a source image to a target image which is then used to warping the source image to obtain an FE-estimated target image, and then the FE-estimated target image is registered to the target image in a subsequent non-rigid image registration process to compensate 
relatively small displacement residuals due to simplifications and uncertainties in the model parameters that are inherent in the biomechanical models. This has the advantages that the deformation recovered by the image registration algorithm is relatively small, reducing the chance of creating physically unrealistic deformation. The accuracy of the proposed method was tested using publicly available annotated 4D CT datasets of 10 lung cancer patients from the DIR-lab database (www.dir-lab.com) (Castillo et al., 2009). The effects of FE model parameters on the accuracy of biomechanical modelling in lung motion estimation and the potential of the pattern analysis of displacement compensation in optimising biomechanical models were also investigated.

The main contributions of the study are in (1) having developed a biomechanical model based non-rigid image registration method that not only can have a comparable registration performance to the state-of-the-art non-rigid intensity-based image registration methods but also can provide physically realistic deformation estimations with an integrated solution for various physical and physiological properties modelling of the lungs in one single FE model, and (2) having demonstrated that the proposed method has the potential to be used for guiding the improvement of biomechanical models through analysing the pattern of displacement compensation from the intensity-based non-rigid image registration process.

\section{Methods and Materials}

In this study, we demonstrated and evaluated the proposed registration method through estimating lung motion from the end-exhale to the end-inspiration, that is, determining the transformation/displacement between two images (a source image and a target image), which correspond to the two breath phases, respectively.

\subsection{Hybrid biomechanical model based image registration method}

Figure 1 illustrates the proposed image registration method, which includes two consecutive processes: (1) patient-specific biomechanical modelling of lung motion with an FE model, and (2) intensity-based image registration.

The biomechanical modelling process consists of two main steps: i) construct a patient-specific FE model based on the geometry models extracted from CT images; ii) 

registration process.

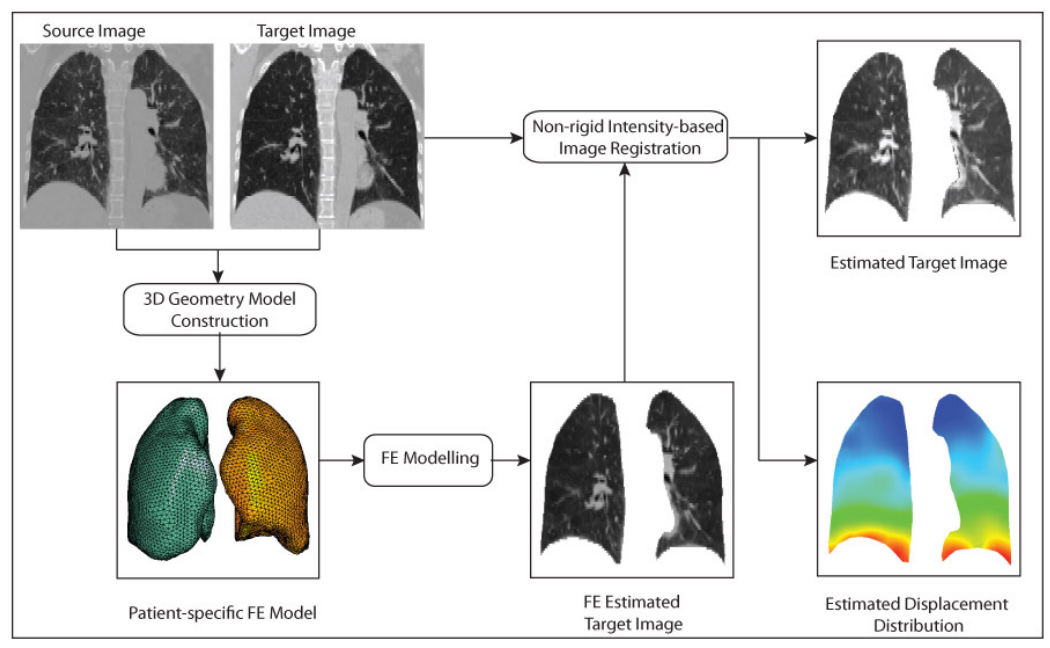

Figure 1: Biomechanical-model based image registration framework

perform biomechanical modelling to estimate displacement fields of the anatomic structures, and then use them to warp the source image to generate an FE-estimated target image. Immediately after the biomechanical modelling process, the intensity-based image registration process is then used to determine the transformations applied to the FE-estimated target image in order to align it with the target image. Since image registration is between the FE-estimated target image and the target image, the transformations obtained are essentially the displacement compensation to the initial FE-estimated displacement field. The estimated total displacement field relating the source image to the target image is now the sum of the FE-estimated displacements from the biomechanical modelling process and the displacements determined from the intensity-based image

\subsection{Biophysical process of lung respiratory motion}

As shown in Fig. 2, the human lungs are situated in the thoracic cavity, with each lung is surrounded by a pleural cavity consisting of two pleurae: the parietal pleura attached to the internal walls of the thoracic cavity (i.e. rib cage), and the visceral pleura covering the surfaces of the lungs. The pleural cavity contains a thin film of pleural fluid, providing lubrication to the parietal and visceral pleurae and allowing them to slide smoothly over one another during respiration. 


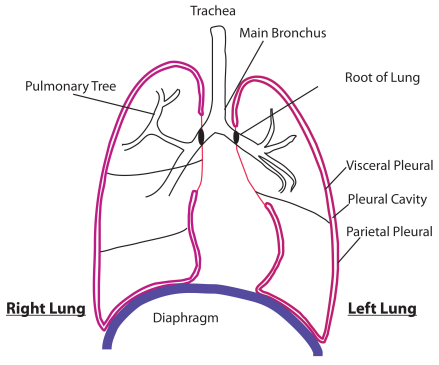

(a)

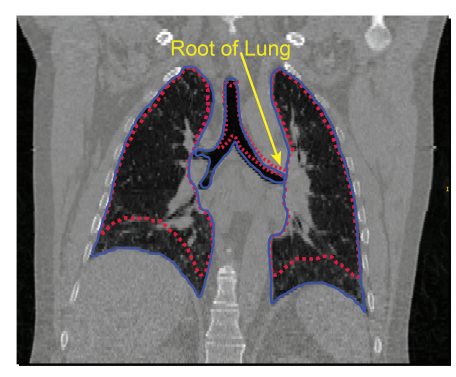

(b)

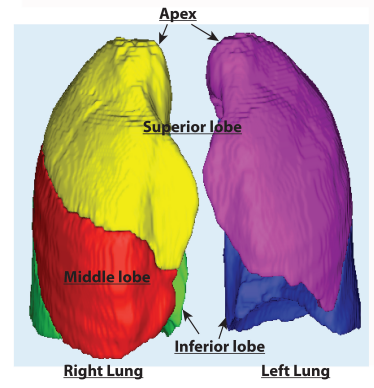

(c)

Figure 2: (a) Schematic diagram of lung anatomy; (b) lung contours at full expiration and full inspiration, superimposed on a coronal slice of a 3D CT volume (Case1); (c) lung lobes segmented from the CT volume. The dashed lines in red and the solid lines in blue shown in Fig. 2(b) correspond to the lung contours at the full expiration and full inspiration, respectively.

The lung is connected to the heart and the trachea by the root of the lung, which is surrounded by pleurae and connects the medial surface of each lung to the heart and trachea (Gray, 1918). The lower end and the bifurcation of the trachea are displaced downwards during inspiration, and the lung expands in a downward and forward direction. The roots of the lungs descend to facilitate this motion. Fig. 2(b) illustrates that the lung roots at full expiration are higher than their positions at full inspiration.

During inspiration, the contraction and the downward movement of the diaphragm cause an increase in thoracic volume and a decrease in pleural and alveolar pressures; consequently, the lungs expand and air is drawn in. During expiration, the relaxation and the upward movement of the diaphragm result in the decrease of thoracic volume and the increase of pleural pressure, the lungs spring back to their original positions and air flows out. The pleural pressure is always negative during normal breathing (Gray, 1918), and the visceral pleura slides against the parietal pleura. Therefore, the respiratory motion of the lung could be modelled by applying a negative pressure to the lung, and the interaction between the lungs and the pleural cavity could be considered as a contact problem (Zhang et al., 2004, Villard et al., 2005, Werner et al., 2009a).

\subsection{Patient-specific biomechanical modelling of lung motion}

Biomechanical modelling for lung motion estimation starts from simulating the lung deformation between two respiratory phases. Under a negative pressure, a deformable 
lung model at its initial state, corresponding to the first respiratory phase, is expanded to its target volume corresponding to the second respiratory phase. The surfaces of the target volume limit the final deformation of the lung model. To generate a patient-specific biomechanical model of a lung, its 3D geometries at the two phases are required. In this study, they were extracted from 4D CT data using a four-step segmentation process as follows:

Step 1. Extract lung regions (including the trachea and airways) using a semi-automatic segmentation method consisting of region competition and level set snake evolution (Yushkevich et al. 2005)

Step 2. Extract the masks of trachea and large airways using the same segmentation process.

Step 3. Remove the masks of trachea and airways from the lung segmentation masks obtained in Step 1 to separate the left and right lungs.

Step 4. Generate closed lung segmentation masks with opening and closing operation.

Normally, the masks of left and right lungs can be separated automatically after Step 3. If the junctions between them are thin and have weak intensity contrast within the images, the separation of the left and right lungs can be performed manually. This is the case for Case 2 and Case 8. The lung volumes following segmentation are summarized in Table 1 .

After lungs have been segmented, an automatic FE model generation process (Han et al. 2012) is used to generate an FE model. A previous study (Amelon, 2012) has shown that the accuracy of FE simulations was not affected by including intra-lobar sliding for 4D CT image registration due to relatively small volume changes of the lungs during free breathing. Therefore, in this study, we only consider the sliding motion between the entire lung and the chest wall. The FE model of the lungs (Han et al., 2014a) includes the deformable lungs extracted from the lung segmentation of 3D CT images at end exhale, and the rigid surfaces extracted from the lung segmentation of 3D CT images at full-inspiration. First-order linear interpolation tetrahedral and second-order quadratic elements are two basic types of elements commonly used for lung motion modelling, a previous study (Al-Mayah et al., 2011) has shown little difference between the models of the two types of tetrahedral elements on lung motion simulations. In this study, the 
Table 1: Lung Volumes of 10 Patients (in litre)

\begin{tabular}{ccccccc}
\hline $\begin{array}{c}\text { Subject } \\
\text { No }\end{array}$ & \multicolumn{2}{c}{$\begin{array}{c}\text { Volume at full } \\
\text { expiration }(l)\end{array}$} & \multicolumn{2}{c}{$\begin{array}{c}\text { Volume at full } \\
\text { inspiration }(l)\end{array}$} & \multicolumn{2}{c}{$\begin{array}{c}\text { Volume } \\
\text { ratio(\%) }\end{array}$} \\
\cline { 2 - 7 } left & right & left & right & left & right \\
\hline Case 1 & 0.95 & 1.26 & 1.00 & 1.37 & 5.46 & 9.15 \\
Case 2 & 2.32 & 2.68 & 2.58 & 2.96 & 10.91 & 10.33 \\
Case 3 & 1.73 & 1.98 & 1.94 & 2.25 & 11.97 & 13.14 \\
Case 4 & 1.14 & 1.53 & 1.32 & 1.74 & 16.02 & 13.65 \\
Case 5 & 1.41 & 1.72 & 1.55 & 1.93 & 9.94 & 12.60 \\
Case 6 & 1.12 & 1.36 & 1.40 & 1.76 & 24.31 & 29.96 \\
Case 7 & 1.40 & 1.70 & 1.73 & 2.07 & 23.23 & 21.38 \\
Case 8 & 2.25 & 2.46 & 2.66 & 3.02 & 18.00 & 22.43 \\
Case 9 & 0.76 & 0.93 & 0.90 & 1.08 & 17.41 & 16.27 \\
Case 10 & 1.16 & 1.76 & 1.36 & 2.04 & 17.71 & 15.89 \\
\hline Mean(SD) & $1.42(0.5)$ & $1.74(0.5)$ & $1.64(0.6)$ & $2.02(0.6)$ & $15.5(5.9)$ & $16.5(6.4)$ \\
\hline
\end{tabular}

deformable lungs are meshed with 4-node tetrahedron elements and the rigid surfaces are meshed with 3-node triangular shell elements. The rigid surfaces are used as constraints to limit the deformation of the deformable lungs, and all 6 degrees of freedoms (DOFs) of the nodes on the rigid surfaces are fixed to prevent rigid-body motions. To simulate the sliding motion of the pleurae against the chest wall, contact pairs are defined between the surface of the deformable lungs and the rigid surfaces, with or without friction.

The lung parenchyma is assumed to be a compressible, non-linearly elastic, homogeneous continuum, modelled with a two-parameter Neo-Hookean model. The strain energy function for describing the Neo-Hookean model, $W$, is defined as

$$
W=C_{10}\left(\bar{I}_{1}-3\right)+\frac{1}{D_{1}}\left(J^{e l}-1\right)^{2}
$$

where $\bar{I}_{1}$ is the first deviatoric strain invariant associated with deviatoric stretches, $J^{e l}$ is the elastic Jacobian, and $C_{10}$ and $D_{1}$ are two material parameters which are related to initial shear modulus, $\mu_{0}$, and initial bulk modulus, $K_{0}$, at small strain, by the relations: 


$$
\mu_{0}=2 C_{10}, K_{0}=\frac{2}{D_{1}}
$$

305

where $\mu_{0}$, and $K_{0}$ are related to two commonly used infinitesimal elasticity parameters, Young's modulus, $E$, and Poisson's ratio, $v$, through the relations:

$$
\mu_{0}=\frac{E}{2(1+v)}
$$

$$
K_{0}=\frac{E}{3(1-2 v)}
$$

Thus, the NeoHookean hyperelastic model can be defined by Young's modulus and Poisson's ratio. Due to a lack of in vivo data on mechanical properties of lungs, different values ranging from $0.1 \mathrm{kPa}$ to $7.8 \mathrm{kPa}$ for Young's modulus, and from 0.2 to 0.45 for the Poisson's ratio, based on in vitro experimental data from dog or human or arbitrary choices, have been used in previous lung studies (Werner et al., 2009a).

In this study, we assume that lungs are homogeneous in the sense that there is no difference in mechanical properties between different lobes (unless otherwise specifically stated). Because of the final shape constraint and the homogeneity assumption, the changes of material parameters (Young's modulus and Poisson's ratio) have little effect on displacement distribution after the lung is expanded to its target volume (Werner et al. 2009a); they only affect the value of pleural pressure required to fully inflate the lung to the target volume. A stiffer lung tissue and a higher value of Poisson's ratio require a higher pleural pressure and a longer computation time if implicit integration schemes are used in the FE modelling. Based on literature values, a reference value of 5 $\mathrm{kPa}$ and 0.2 are chosen for Young's modulus and Poisson's ratio, respectively.

With the specified material parameters, the minimum pleural pressure required to expand a lung to its target volume could be estimated from the definition of bulk modulus (Villard et al. 2005). Bulk modulus, $K$, is a measure of the substance's resistance to uniform compression defined as the ratio of the infinitesimal pressure increase to the resulting relative decrease of the volume.

$$
K=-V_{0} \times \frac{\mathrm{d} P}{\mathrm{~d} V}
$$


where $V_{0}$ is the initial volume of the lung, $\mathrm{d} P$ and $\mathrm{d} V$ are the difference in pleural pressure and the difference in lung volume at two different breathing phases, respectively. The inverse of the bulk modulus gives the lung's compressibility, which can be expressed as:

$$
\frac{1}{K}=-\frac{1}{V_{0}} \times \frac{\mathrm{d} V}{\mathrm{~d} P}
$$

where $\mathrm{d} V / \mathrm{d} P$ is known as the pulmonary compliance - a measure of how easy it is to inflate, which can be obtained by analysing the pressure-volume curve of the lung. Low compliance indicates a stiff lung and means extra work is required to bring in a normal volume of air. This occurs as the lungs in this case become fibrotic, lose their dispensability and become stiffer. On the other hand, patients with a high lung compliance due to the poor elastic recoil have no problem inflating the lung but have difficulty exhaling air (Galetke et al., 2007). Combining Eqs.(4) and (6), we have

$$
\frac{\mathrm{d} P}{E}=-\frac{1}{3(1-2 v)} \frac{\mathrm{d} V}{V_{0}}
$$

where the initial lung volume, $V_{0}$, and its volume change, $\mathrm{d} P$, can be obtained from 4D CT segmentation summarised in Table 1. However, Eq. 77 is only valid for a free expansion of the lungs under a uniform pressure, which may underestimate the minimum required pleural pressure (Werner et al. 2009a). Because of the effect of contact interaction between the deformable lung and the rib cage, the minimum required value of pleural pressure could be much higher. As can be observed in Table 1, Case 6 has the maximum volume change ratio of 0.3 , thus the minimum pleural pressure estimated from Eq. (7) is $0.83 \mathrm{kPa}$. If we define the success criteria for FE simulations as the volume of the deformed lung is $\geqslant 99.5 \%$ of its final target volume, then this value is too small for FE simulations. In this study, we found a pressure load of $3 \mathrm{kPa}$ was large enough to deform a lung to $99.5 \%$ of its target volume in FE simulations for all 10 cases. All simulations were performed with a nonlinear implicit procedure available in the commercial nonlinear implicit FE solver, ABAQUS/standard, with geometrical nonlinearity included for large deformation analyses.

\subsection{Intensity-based non-rigid image registration}

In principle, any non-rigid image registration can be integrated into the proposed registration scheme. Many different transformation models are available and we refer inter- 
ested readers to extensive reviews on medical image registration (Maintz and Viergever 1998, Holden, 2008; Sotiras et al., 2013) for further information. The choice of registration method depends on anatomic structures of interest, as well as clinical applications and constraints. In this study, we attempt to make full use of intensity information in medical images to provide an accurate registration for internal structures and features. Therefore, we chose to focus on intensity-based, non-rigid registration schemes, implemented using popular B-spline transformation models (Rueckert et al., 1999 Klein et al. 2010) (see details in Section 3).

\subsection{Evaluation of the proposed algorithm}

Dynamic lung images in particular have been used widely for evaluating deformable image registration algorithms. To evaluate the proposed method and facilitate the comparison with other registration methods in the literature, we performed intra-patient non-rigid registration of 3D CT data drawn from lung cancer patient 4D CT datasets in the DIR-Lab database (www.dir-lab.com) (Castillo et al., 2010). These datasets have already been used for validating and evaluating different registration methods of sliding objects in several publications (Schmidt-Richberg et al., 2012b; Delmon et al., 2013 Heinrich et al., 2013, Fuerst et al., 2015). In these datasets, each 4D CT scan includes ten 3D CT images obtained over a breathing cycle. The slice thickness of each 3D CT image is $2.5 \mathrm{~mm}$ and the in-plane spatial resolution ranges from $0.97 \mathrm{~mm} \times 0.97 \mathrm{~mm}$ to $1.16 \mathrm{~mm} \times 1.16 \mathrm{~mm}$. Each 3D CT scan comes with a set of 300 inner-lung landmarks, carefully annotated by experts. Thus, a total of 3000 internal landmarks are available. The intraobserver variants of the 10 cases range from $0.70(0.99) \mathrm{mm}$ to $1.13(1.27) \mathrm{mm}$, with an average of $0.88(1.3) \mathrm{mm}$. The uncertainty in the landmark selection is within the voxel size of the images. The lung volumes of the 10 patients estimated from the lung segmentation process described in Section 2.3 are listed in Table 1. These values indicate that the volumes and the expansion rates of lungs during a full breathing cycle vary significantly between individuals, from $5.5 \%$ to $30 \%$ (Note also that the left lung is slightly smaller than the right lung in each case).

In this study, we used image pairs consisting of 3D CT images of lungs at the end of inspiration and their corresponding images at the end of expiration to evaluate the registration accuracy, in terms of an anatomical-landmark-based target registration error 
(TRE). We particularly analysed the registration error distributions of those landmarks within an inner region of $10 \mathrm{~mm}$ near lung surfaces, where the accuracy of intensity-based image registration methods is mostly affected by sliding motion. The effects of parameters in biomechanical models on displacement estimation have also been investigated.

\section{Results}

To quantify the registration accuracy of the proposed method and investigate the effects of model parameters, we calculated the target registration error (TRE) defined as the Euclidean distance between 300 pairs of internal anatomical landmarks which are provided with the DIR-lab dataset and identified in the target image and transformed source image space for each case. Furthermore, we compared the proposed method, referred here to as $\mathbf{F E}+\mathbf{B}$-spline, with a biomechanical simulation method, a conventional non-rigid B-spline registration without a consideration of sliding motion (Klein et al. 2010), and two alternative non-rigid intensity-based image registration methods with a specific handling of sliding motion.

The four methods used for the purposes of comparison are summarised as follows

- Method 1: Biomechanical simulation (Werner et al., 2009a|b), (identical to that used in the first process of the proposed method).

- Method 2: Separate image registration of the lungs and other anatomy using conventional B-spline registration and lung masks (Wu et al., 2008).

- Method 3: Image registration with sliding regularisation based on directiondependent B-spline decompositions (Vandemeulebroucke et al., 2012: Delmon et al. 2013).

- Method 4: Conventional B-spline registration without special considerations for sliding motion (Klein et al. 2010).

Since the Elastix toolbox for intensity-based image registration (Klein et al., 2010) (http:/elastix.isi.uu.nl), has implemented conventional B-spline transformation models being used for Method 2 and Method 4, and sliding motion regularisation scheme used in Method 3, it was used in this study. To ensure a fair comparison, the same 


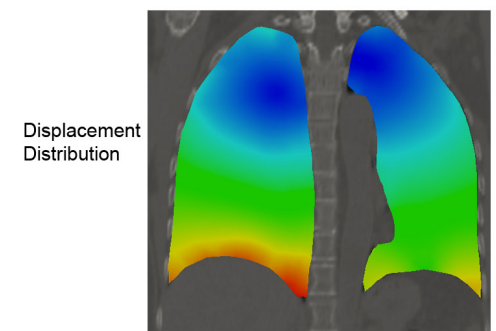

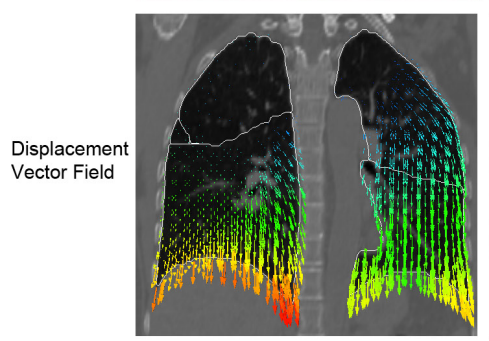

(a) FE Simulation
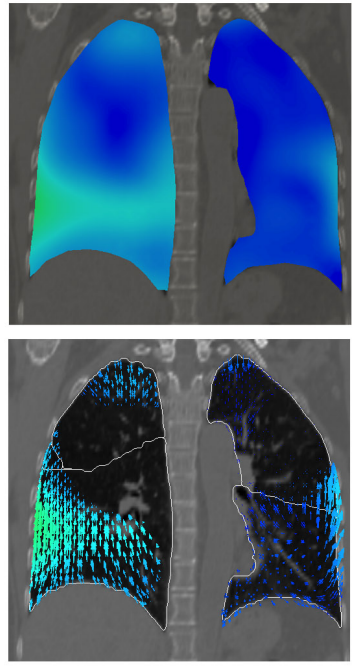

(b) Displacement Compensation
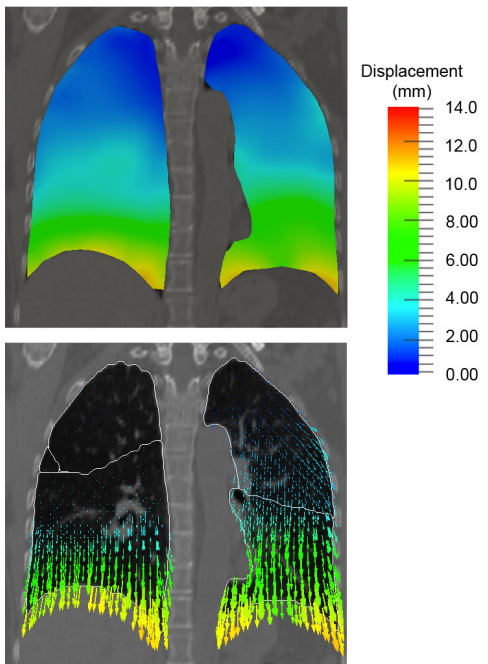

(c) Final Result

Figure 3: Displacement field estimation for Case 1 from two consecutive processes of the proposed method during registration: (a) FE estimated displacement field (b) displacement compensation from the subsequent B-spline registration (c) final displacement field. In the FE simulations, the lungs were assumed to be homogeneous and frictionless against the chest wall. The displacement magnitude ranges from $0.28 \mathrm{~mm}$ to $13.6 \mathrm{~mm}$.

420

\subsection{Displacement evolution during registration}

Figure 3 illustrates a typical example of displacement evolution during the two-process registration of our method. The deformed lung models are overlaid on the original CT image at full inspiration. Both the displacement magnitudes and the directions are plotted. Fig. 3(a) shows a 2D displacement distribution from biomechanical modelling in 
the coronal plane for Case 1. In the biomechanical simulations, we assumed that the two lungs were homogeneous and the interaction between the lungs and the chest wall was frictionless. Fig. 3(b) is the displacement compensation to biomechanical modelling by the subsequent non-rigid B-spline registration process, and Fig. 3 (c) is the total displacement distribution after the two-process registration. As can be seen in Fig. 3(a), the FE model captures a substantial part of the lung deformation, and the subsequent B-spline registration compensates for relatively small residuals $(<5.0 \mathrm{~mm})$ distributed in the right lower lobe (Fig. $3(\mathrm{~b})$ ).

\subsection{Influence of material parameters}

To investigate the effect of Poisson's ratio, we used Case 1 as an exemplar and plotted the volume ratio between the deformed volume and its target volume against the applied dimensionless pleural pressure, represented as, $\mathrm{d} P / \mathrm{d} E$, for different values of Poisson's ratio $v=(0.1,0.2,0.3,0.4$ and 0.45$)$ (see Fig. 4). Inspection of Fig. 4 reveals that the higher the value of Poisson's ratio, the higher the pleural pressure required to deform the initial lung volume to its target volume. When different values of Poisson's ratio are assigned, the volume change follows different paths before finally reaching a plateau close to 1.0. Therefore, we may conclude that the choice for the value of Poisson's ratio does affect biomechanical modelling process of lung motion.

In addition, we investigated the effect of Poisson's ratio on the final deformation field following a successful FE simulation, that is, when the plateau shown in Fig. 4 is reached. Figure 5 illustrates the displacement distributions during the registration processes for different values of Poisson's ratio. Both FE simulation results and final registration results using the proposed method are presented. The pattern of the final displacement fields does not show significant difference between the four FE models with different values of Poisson's ratio. After FE simulations, the mean (the standard deviation (SD)) of the target registration errors (TRE) of 300 lung landmarks for the four FE models were $1.80(0.95) \mathrm{mm}, 1.79(0.99) \mathrm{mm}, 1.77(0.92) \mathrm{mm}, 1.81(1.13) \mathrm{mm}$, respectively. After final registration using the proposed method, the final mean (SD) of the TRE was reduced to 1.06(0.54) $\mathrm{mm}, 1.08(0.54) \mathrm{mm}, 1.08(0.55) \mathrm{mm}$ and 1.09(0.54) $\mathrm{mm}$, respectively. Therefore, in terms of TREs of landmarks, the choice of Poisson's ratio does not have an obvious impact on either the final FE simulation results after the lungs are expanded above $99.5 \%$ 


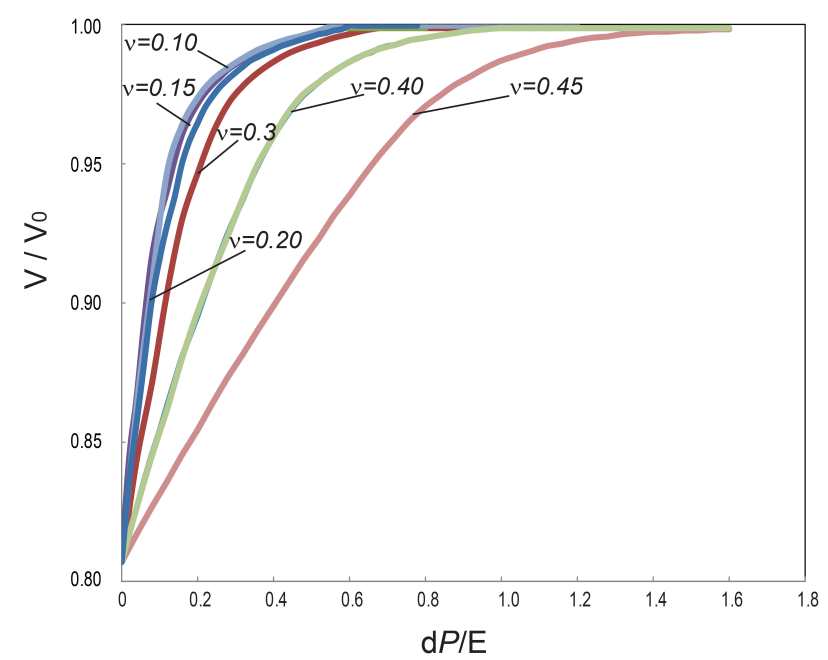

Figure 4: Relationships between the increase of pleural pressure and the ratio of the deformed lung volume to its target volume for different values of Poisson's ratio of lung tissues.

of their target volumes or on the final registration results.

\subsection{Effect of friction}

For a normal lung, it is expected that the lung and the pleural cavity slide against each other smoothly. However, there may exist small friction on the sliding interface due to the existence of lung diseases or tumours near lung surfaces. To investigate the friction effect on FE simulations and final registration accuracy, a frictional contact model is used for patient-specific biomechanical modelling. We used a default setting of ABAQUS with a penalty friction formulation for contact analysis, and chose four different values $\alpha=(0.05,0.1,0.2$ and 0.3$)$ for the friction coefficient, $\alpha$. For the sake of simplicity, the same parameters were used for both the left and the right lung models. The effect of friction on displacement distributions during the proposed registration processes was analysed. Figure 6 shows a comparison of displacement distributions during the registration processes between a frictionless model and four frictional models. The displacements obtained from FE simulations, the displacement compensations of B-spline registration, and the combined results are presented as well.

As shown in Fig. 6 (first row), FE simulation results of displacement distribution 


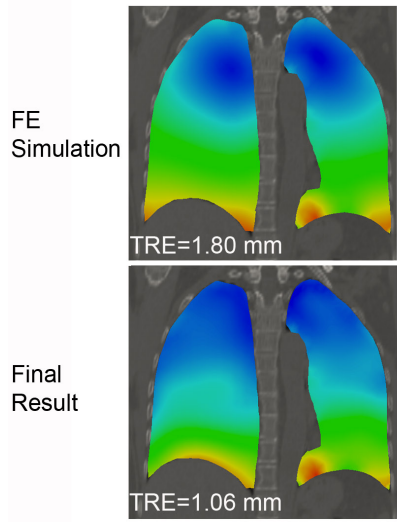

(a) $\nu=0.1$

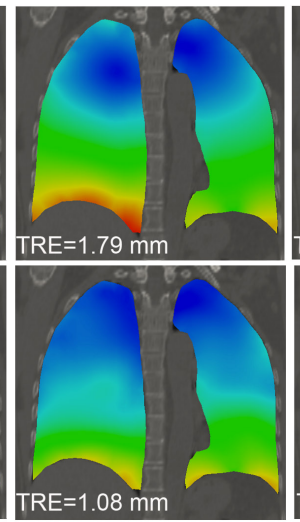

(b) $\nu=0.2$
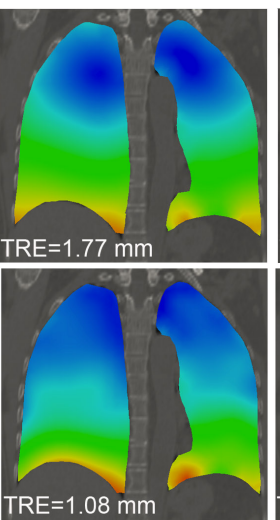

(c) $\nu=0.3$

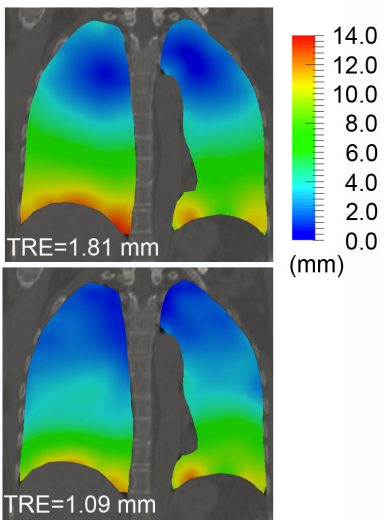

(d) $\nu=0.4$

Figure 5: Displacement field distributions of the lungs from FE simulations using four different values of Poisson's ratio after the lung models are expanded to their target. The first row shows FE simulation results; the second row shows final registration results using the proposed method. The colour denotes the displacement magnitude increasing from blue to red.

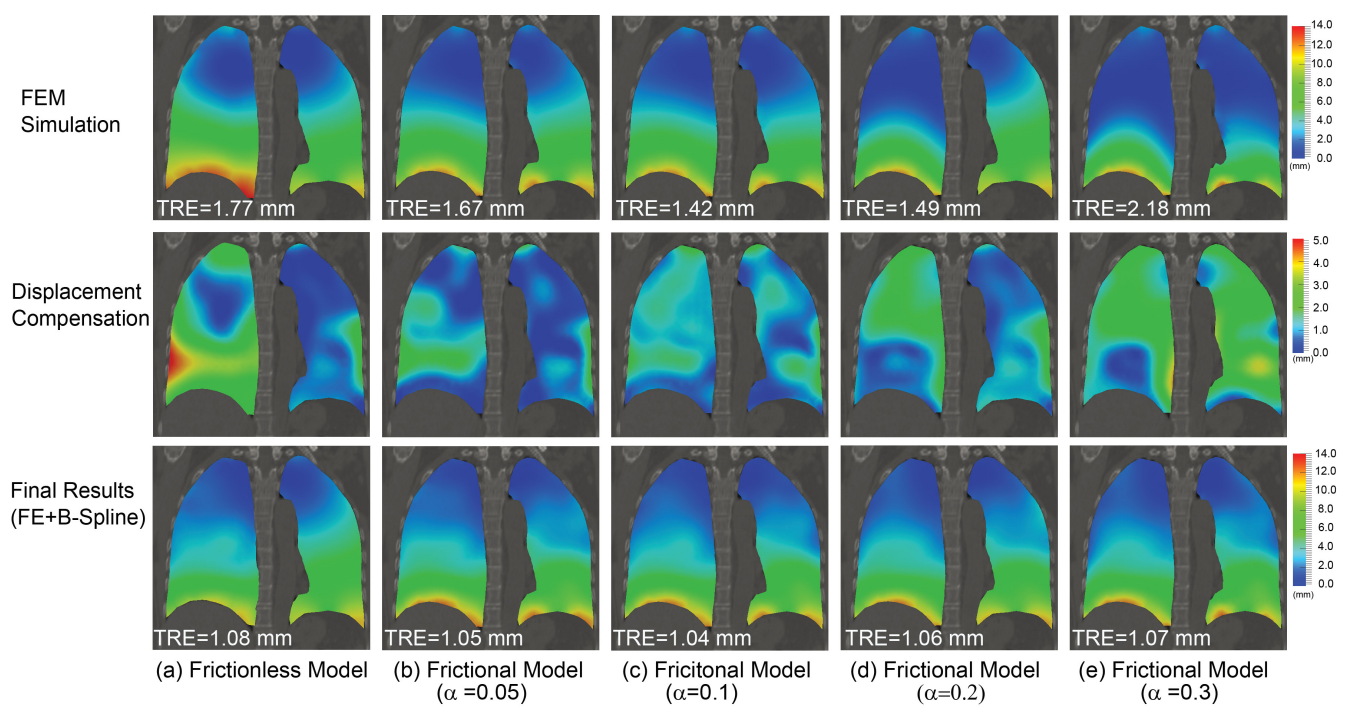

Figure 6: Friction effect on finite element simulation results of displacement distribution and final registration accuracy for Case 1. The distributions of displacement magnitude are superimposed on the 2D coronal slice of CT images at full inspiration. The results of a frictionless FE model (a) are compared with those of frictional FE models with different values of friction coefficient (b) 0.05 , (c) 0.1 (d) 0.2 and (e) 0.3 . The colour denotes the displacement magnitude increasing from blue to red. 
are different when different friction coefficients are used in FE models. The pattern of displacement distributions shows that the motion of the upper lobes of the lungs is reduced with the increase in the coefficient of friction. The mean (SD) TREs of 300 landmarks are also affected: $1.77(0.99) \mathrm{mm}$ for frictionless case, $1.67(0.71) \mathrm{mm}$ for $\alpha=$ $0.05,1.42(0.67) \mathrm{mm}$ for $\alpha=0.1,1.49(0.77) \mathrm{mm}$ for $\alpha=0.2$ and 2.18(1.14) $\mathrm{mm}$ for $\alpha=$ $0.3)$, respectively. The FE model with a friction coefficient of 0.1 gives the best prediction on the displacements of the landmarks, although other choices on friction coefficient below $\alpha=0.3$ produce similar accuracies. The final registration results are presented on the third row of Fig. 6. The distribution of total displacements shows no difference when different frictional models are used, this is further confirmed by very small TRE differences of the landmarks with the combined method. The mean (SD) TREs of the 300 landmarks are 1.08(0.5) $\mathrm{mm}$ (frictionless), 1.05(0.55) $\mathrm{mm}(\alpha=0.05), 1.04(0.54) \mathrm{mm}$ $(\alpha=0.1), 1.06(0.53) \mathrm{mm}(\alpha=0.2)$ and 1.07(0.54) $\mathrm{mm}(\alpha=0.3)$, respectively.

Although FE models with different friction coefficient values produce different displacement estimations, the proposed registration method provides the same registration accuracy for all models, thanks to the displacement compensation to FE simulations in the subsequent intensity-based registration. The displacement compensations to FE models are plotted on the second row of Fig. 6. The larger displacement compensations to the FE simulations on the right lower lobes close to the chest wall indicate that the frictionless model may overestimate the displacement of right lower lobes. By introducing a frictional contact model to limit the motion of the lower lobe near to the chest wall, the overestimated displacement could be partially compensated. For instance, the mean TRE was reduced from $1.77 \mathrm{~mm}$ to $1.42 \mathrm{~mm}$ by introducing a small amount of friction (e.g. $\alpha=0.1$, Fig. 6(c)). However, introducing too much friction (e.g. $\alpha=0.3$, Fig. 6(d) may significantly over-constrain the deformation of the upper lobes, causing an underestimation of the amount of deformation.

\subsection{Effect of tissue heterogeneity}

In reality, the tissue distributions of lungs are not homogeneous, but utilising a heterogeneous tissue model can significantly increase the complexity of modelling. To investigate the effect of tissue homogeneity assumption on FE simulations and registration accuracy, we performed an experiment on Case 8, which has the highest mean TRE er- 
ror of $15 \mathrm{~mm}$ for 300 landmarks among all 10 datasets before registration, as shown in Table 2

3D CT images of Case 8 revealed a small tumour in the left upper lobe, as shown in the coronal slice on the second column of Fig. 7, which might severely increase the stiffness in this region and in turn affects the deformation characteristics. Therefore, we proposed a heterogeneous FE model to account for the difference in stiffness for each lobe. The lobes of both lungs were segmented manually using the same process described in (Han et al. 2014b) and different Young's moduli were assigned to different lobes. For the purposes of testing, we assumed that the right lower lobe was softer than both the right middle and right upper lobes, whilst the left upper lobe was harder than the left lower lobe due to the existence of a tumour. In the FE model, the right lower lobe was assigned with a Young's modulus of $2.5 \mathrm{kPa}$; the left upper lobe was assigned with a larger value of Young's modulus, $10 \mathrm{kPa}$; and all the other lobes were assigned with a Young's modulus of $5 \mathrm{kPa}$. The choice of Young's moduli for soft/hard lobes was arbitrary and only for the purpose of demonstrating the effect of tissue heterogeneity.

Figure 7 shows the change in displacement distribution during registration when a homogeneous tissue model is replaced by a heterogeneous tissue model in the biomechanical modelling process. Frictionless contact is assumed for both models. The distribution of displacement compensation (the second column in Fig. 7) shows that the homogeneous tissue model may underestimate the deformations of both the left upper lobe and the right lower lobe. This may be caused by the difference of each lobe in mechanical properties or the non-uniform pleural pressure between different lobes (Permutt et al., 1962 , West et al., 1964). In particular, the 3D CT images of Case 8 revealed a small tumour in the left upper lobe (coronal slice on the second column of Fig. 7), which might severely increase the stiffness in this region and in turn affects the deformation characteristics. Therefore, we proposed a heterogeneous FE model to account for the difference in stiffness for each lobe. The lobes of both lungs were segmented manually using the same process described in (Han et al., 2014b) and different Young's moduli were assigned to different lobes. Figure 7 (b) shows the result of displacement distribution when an FE model with a heterogeneous distribution of tissues is used. For the purposes of testing, we assumed that the right lower lobe was softer than both the right middle and right 
(a)

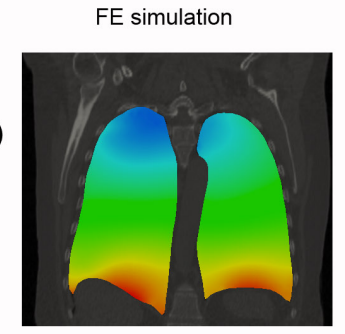

(b)

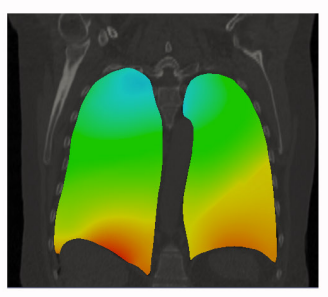

Displacement Compensation
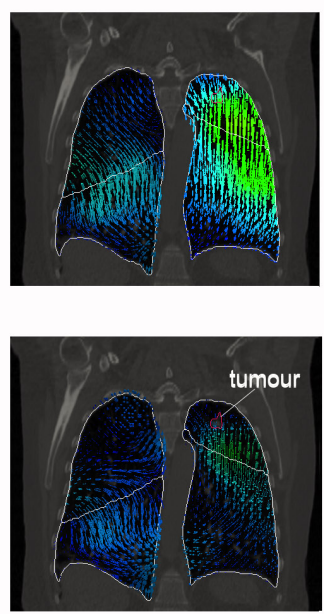

Final Results
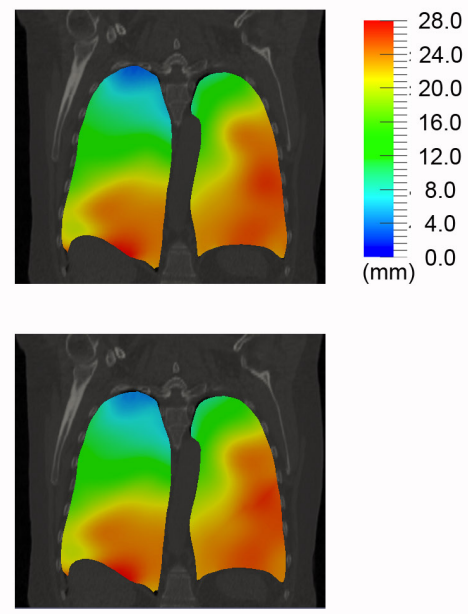

Figure 7: A comparison of displacement distributions during the two-process registration between (a) a homogeneous model and (b) a heterogeneous model.

Since biomechanical models can explicitly include physical properties (such as stiffness) of tumours and characterise their motion behaviour during respiration, it is ex- 
pected that introducing the tissue heterogeneity in the biomechanical modelling process could improve the performance of the hybrid registration method on physically realistic deformation/motion estimation of tumours. For example, there exists a tumour located in the lower lobe of the Patient's left lung in Case 6, as shown in Fig. 8(a). The volume of the tumour does not show an observable change on 4D CT images. Therefore, in the FE model, we assume that the tumour has a Young's modulus of $25 \mathrm{kPa}$, five times stiffer than its surrounding tissues. In such a way, we expect that the final volume change of the tumour in the proposed registration method is small. Our method was compared with two non-rigid B-spline registrations with a specific consideration of sliding motion: Method 2 (Wu et al., 2008) and Method 3 (Delmon et al., 2013), outlined above. Figure 8 presents the maps of estimated lung volume changes with the three methods for Case 6. It shows that the two intensity-based B-spline transformations fail to preserve the volume of the tumour, although all three methods produce the same pattern of volume change and the similar registration errors for Case 6. Another non-rigid image registration method that can cope with the sliding motion, MRF-based deformable registration (Heinrich et al. 2013), also did not provide sufficient volume preservation of the tumour for Case 6 (see Fig.3 in the reference (Heinrich et al. 2013)). Although the volume preservation can be kept under the framework of intensity-based image registration methods, e.g. with a tissue-dependent filtering method (Staring et al., 2007), it is much easier for our method to preserve the tumour volume by directly including tumour-specific physical data, such as stiffness which may be measured from elasticity imaging/biopsy, into biomechanical models. The results demonstrates that the proposed registration method has an advantage in volume preservation relevant to the scenario where a hard tumour exists

\subsection{Quantitative comparison and evaluation}

As stated above, the proposed algorithm was evaluated quantitatively by calculating the TREs for 300 internal lung landmarks for each case. Our method, FE+B-spline, was compared with four methods, Methods 1-4 outlined above. Table 2 summarises the mean(SD) TREs over 300 landmarks for each lung cancer patient and for each of the five registration methods. The results show that biomechanical modelling (Method 1), could achieve better registration accuracy $($ mean $\mathrm{TRE}=3.81 \mathrm{~mm})$ than Method $4(\operatorname{mean}$ 


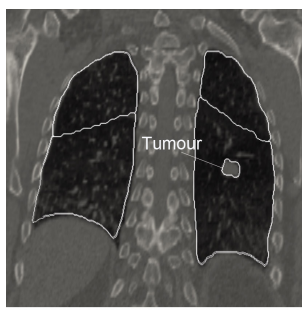

(a) Coronal Slice

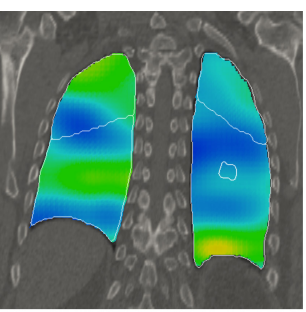

(b) Method 2

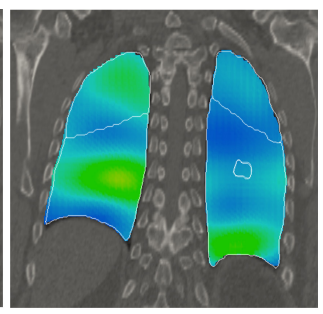

(c) Method 3

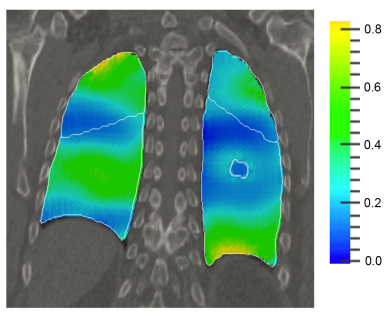

(d) FE+B-Spline

Figure 8: A comparison of volume change ratio for Case 6 with three methods: (b) Method 2: Separate image registration based on B-splie and lung masks, (c) Method 3: B-spline image registration with sliding regularisation, and (d) our method $(\mathrm{FE}+\mathrm{B}-$ spline). Fig. 8(a) is a 2D coronal slice superimposed with a contour of a hard tumour and the contours of lung lobes. Although all of the three methods produce the same pattern of volume increase, our method can explicitly include the stiffness information of the tumour to ensure volume preservation.

$\mathrm{TRE}=4.57 \mathrm{~mm}$ ), even if a simple homogeneous FE model is used. Our method has achieved a registration accuracy comparable to Method 2 and Method 3, both of them consider the effect of sliding motion.

To compare the five registration methods in terms of registration error distribution, we calculated both accumulated and frequency distributions of TRE for all 3000 landmarks of 10 cases (300 landmarks per patient) for each method. In addition, we evaluated the registration error distribution, using the landmarks near the surface of the lungs where sliding occurs, defined as the landmarks lying within an inner region of $10 \mathrm{~mm}$ near the surface. Accurate registration on surface regions is particularly important for the accurate dose accumulation in the radiotherapy and HIFU (high intensity focused ultrasound) ablation of tumours, such as non-small cell lung cancer, adenocarcinoma, large cell carcinoma, and pleural mesothelioma covering on the lung surface (Muers, 2003). Of the 3000 internal landmarks available, 554 were located in the near-surface region. The corresponding registration results are presented in Figs. 9 .

As shown in Fig. 9, our method produces the lowest registration error in terms of cumulative distributions of the TREs. When all the landmarks are taken into account, numerically, the proposed method is superior to Method 3 and slightly better than Method 2. The mean (SD) TREs of Methods 1-5 are 3.81(2.65) mm, 1.45(0.99) mm, $1.71(1.31) \mathrm{mm}, 4.57(5.32) \mathrm{mm}$ and $1.37(0.89) \mathrm{mm}$, as listed in Table 2 . 
Table 2: Registration Results of Five Registration Methods (Mean TRE(SD) in $m m$ ). The five methods are Method 1: Biomechanical simulation; Method 2: Separate image registration based on B-spline and lung masks; Method 3: B-spline image registration with sliding regularisation; Method 4: Conventional B-spline registration; and Our Method (FE+B-Spline), respectively. The calculation of mean TRE(SD) uses all 300 landmarks for each subject.

\begin{tabular}{ccccccc}
\hline Patient & $\begin{array}{c}\text { Before } \\
\text { Registration }\end{array}$ & & & & & \\
\multicolumn{1}{c}{ Method 1 } & Method 2 & Method 3 & Method 4 & $\begin{array}{c}\text { Our Method } \\
\text { (FE+B-Spline) }\end{array}$ \\
\hline 1 & $3.89(2.78)$ & $1.77(0.92)$ & $1.14(0.64)$ & $1.21(0.52)$ & $1.63(1.09)$ & $1.08(0.55)$ \\
2 & $4.34(3.90)$ & $2.14(1.28)$ & $1.03(0.50)$ & $1.06(0.52)$ & $1.85(1.88)$ & $0.99(0.49)$ \\
3 & $6.94(4.05)$ & $3.90(2.10)$ & $1.28(0.67)$ & $1.83(1.02)$ & $3.26(2.47)$ & $1.22(0.65)$ \\
4 & $9.83(4.85)$ & $4.04(2.21)$ & $1.50(1.01)$ & $1.71(1.09)$ & $3.34(2.85)$ & $1.49(0.99)$ \\
5 & $7.48(5.50)$ & $3.39(2.17)$ & $1.88(1.41)$ & $1.94(1.54)$ & $4.18(3.80)$ & $1.73(1.38)$ \\
6 & $10.9(6.96)$ & $3.54(2.23)$ & $1.52(0.87)$ & $1.70(0.94)$ & $5.10(4.46)$ & $1.48(0.86)$ \\
7 & $11.0(7.42)$ & $4.22(2.91)$ & $1.61(1.09)$ & $1.98(1.30)$ & $7.07(6.42)$ & $1.50(0.85)$ \\
8 & $15.0(9.00)$ & $6.95(3.61)$ & $1.49(1.13)$ & $2.41(2.45)$ & $10.88(9.63)$ & $1.48(1.05)$ \\
9 & $7.9(3.97)$ & $4.26(1.91)$ & $1.40(0.76)$ & $1.56(0.86)$ & $4.32(2.94)$ & $1.38(0.71)$ \\
10 & $7.3(6.34)$ & $3.89(2.46)$ & $1.51(1.08)$ & $1.71(1.22)$ & $4.07(4.66)$ & $1.41(0.84)$ \\
\hline Mean(SD) & $8.46(5.62)$ & $3.81(2.65)$ & $1.45(0.99)$ & $1.71(1.31)$ & $4.57(5.32)$ & $1.37(0.89)$ \\
\hline
\end{tabular}




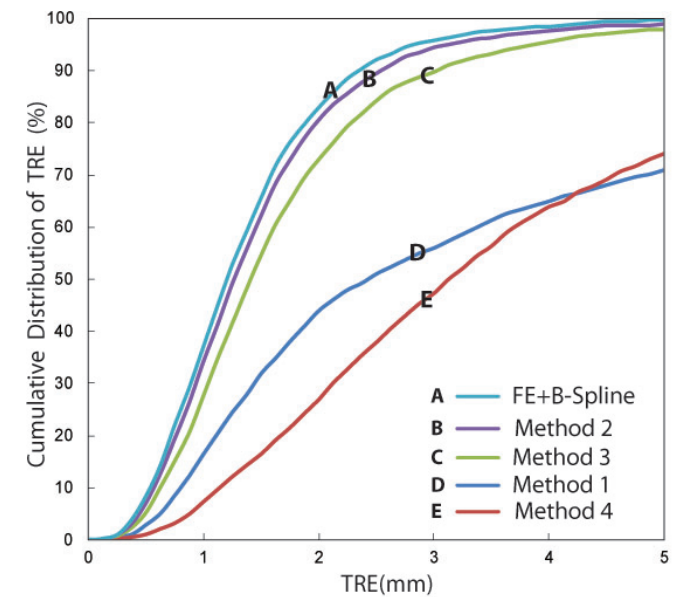

(a) 3000 landmarks

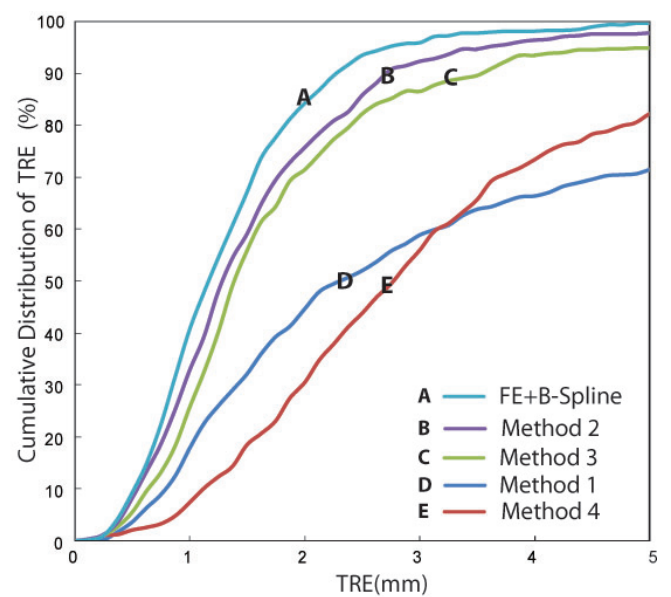

(b) 554 landmarks close to surface

Figure 9: Cumulative distributions of the TRE of landmarks for 10 subjects (Case1Case10): (a) all of 3000 landmarks (2) 554 landmarks near the lung surface within a $10 \mathrm{~mm}$ depth. Five registration methods are compared, including (A) Our method: FE+B-spline, (B) Method 2: Separate image registraiton based on B-spline and lung masks, (C) Method 3: B-spline with sliding regularisation, (D) Method 4: Conventional B-spline, and (E) Method 1: Biomechanical simulation.

When only the landmarks near the lung surface are considered, our method also performs better than any other methods(see Fig $9(\mathrm{~b})$ ). The mean (SD) TREs with the five registration methods are $3.35(2.4) \mathrm{mm}, 1.60(2.14) \mathrm{mm}, 1.96(1.35) \mathrm{mm}, 4.9(6.21 \mathrm{~mm})$ and $1.33(0.79) \mathrm{mm}$, respectively. The maximum errors corresponding to the five methods are $20.7 \mathrm{~mm}, 19.1 \mathrm{~mm}, 15.7 \mathrm{~mm}, 24.9 \mathrm{~mm}$ and $5.3 \mathrm{~mm}$, respectively. The number of landmarks with a TRE $>5 \mathrm{~mm}$ is $98,8,28,86$ and 2 for the four methods, respectively. These results suggest that the proposed method provides a better registration accuracy on the near-surface regions in terms of the landmarks.

\section{Discussion}

\subsection{Registration accuracy}

Compared to intensity-based image registration methods, biomechanical models for 4D CT lung motion estimation can explicitly model certain breathing dynamics and provide physically realistic results. However its registration accuracy in terms of landmark errors is overshadowed by intensity-based image registration methods due to a lack of considering the anatomic details. To improve the registration performance and best 
preserve the desired properties of biomechanical modelling, we introduced an intensitybased image registration process to compensate the displacement residuals of biomechanical modelling. The results presented in Section 3 show that the proposed method can significantly reduce the mean TREs of the 10 cases, dropping from $3.81 \mathrm{~mm}$ to $1.37 \mathrm{~mm}$.

The accuracy of the proposed approach compared well with the results of previously published methods on the same datasets, for example, our method (mean TRE= $1.37 \mathrm{~mm})$; non-rigid diffusion registration with direction-dependent regularization for sliding motion (Schmidt-Richberg et al. 2012b) (mean TRE $=2.13 \mathrm{~mm}$, improved to $1.55 \mathrm{~mm}$ in (Schmidt-Richberg et al. 2012a) ); B-spline registration with direction dependent Bsplines decomposition for sliding motion (mean TRE $=1.71 \mathrm{~mm}$ (Delmon et al., 2013)) and Markov random field (MRF)-based deformable registration (mean TRE $=1.52 \mathrm{~mm}$, and $1.43 \mathrm{~mm}$ with a hyper-label for intensity correction and measurement of the density change (Heinrich et al., 2013)). Particularly we evaluated the registration performance of our method on the near-surface regions where the accuracy of intensity-based image registration methods often suffers from the difficulty in handling with the sliding motion of lungs against rib cage and diaphragm. We compared our method with a conventional B-spline based image registration method without a regularisation on sliding motion, and two B-spline based image registration methods with a specific consideration of sliding motion. It was found that our method provided better registration performance, which may be due to explicit modelling on lung sliding with FE models. The performance improvement may be especially important for the cases when there is a need for an accuracy localization of mobile, superficial tumour.

Although our methods performed the best in the accuracy evaluation of algorithms on the 4D CT datasets of 10 lung cancer patients publicly available, we do not expect that the combined approach would definitely result in better results than other recently developed intensity-based image registration algorithms with a specific consideration of sliding motion. Rather, we feel that the combination of both approaches facilitates the handling of various physical and physiological properties modelling through an integrated biomechanical model, helps to improve the registration accuracy near the surface regions, and potentially provides a guide for improving predictive biomechanical models through analysing the pattern of displacement compensation from the intensity-based image reg- 
istration process

\subsection{Biomechanical model and parameters}

In the proposed method, a nonlinear hyperelastic material model was chosen for biomechanical modelling due to large deformation of the lungs. Previous studies AlMayah et al., 2009, Werner et al., 2009a) showed that a hyper-elastic material model produced slightly better prediction results on displacement than a linear model, but the difference between the two models was small if a contact model was included to simulate the pleural sliding. In this study, we found that the change of Poisson's ratio did not show a significant impact on the displacement distribution of FE models after the lung was expanded to more than $99.5 \%$ of its target volume, but it did affect the deformation states of lungs during the loading process. As shown in Fig. 4 the volume change of lungs follows different paths with increasing loading for different values of Poisson's ratio. Therefore, it is critical to choose an optimal value for the Poisson's ratio if biomechanical models are used for the purpose of predicting the motion in all phases of the respiratory cycle rather than only finding the absolute difference in deformation between two phases.

Like most of studies of lung motion with biomechanical modelling, we only considered the sliding motion between the entire lung and the chest wall, and ignored the intra-lobar sliding. This treatment was based on the consideration that the significance of intralobar sliding in the FE model may be limited during free breathing 4D CT imaging. Amelon's PhD study (Amelon, 2012) indicated that the registration accuracy of FE simulations, based on 4D CT lung images, were not improved after introducing frictionless lobar sliding. Moreover, the lobar segmentations on 4D CT images are difficult due to unclear/incomplete fissures on CT images. To our best knowledge, an automatic lobar segmentation of 4D CT lung images does not exist, and the lung lobar segmentation has to be performed manually. Different interpretation of fissure location may have a more significant impact on the FE solution than considering lobar sliding in the FE model. However, lobar sliding may need to be explicitly modelled if volume changes of lungs are large, e.g. during a breath-hold CT scanning, or when understanding the regional lung function is important (Amelon et al. 2014). In contrast to the difficulty of lobar segmentation, the segmentation of entire lungs are relatively easier. In this study, we adopted a semi-automatic method. It is understandable that the segmentation inconsistency will 
affect the accuracy of FE simulations, but its impact is not significant considering that the volume change of an FE model due to segmentation inconsistency is small, compared with the entire volume of a lung. However, the segmentation inconsistency may result in inaccuracy along the boundary of the lungs in image registration, since the image intensity-based cost function will drive the boundaries of the lung segmentations obtained from the two images to match (Wu et al. 2008 ).

In the hybrid method, biomechanical models facilitate the simulations of lung's interactions with the chest wall, which is a big challenge for intensity-based non-rigid image registration methods. For example, during respiratory, pleural integrity and pleural fluid provide a very low friction between the lungs and the chest wall, thus, a frictionless contact may be sufficient to model the sliding motion, as we did in this study. If the lubrication condition in the pleural cavity changes due to a lung disease, such as pleural adhesion or pleural effusion, a frictional contact could be defined for the contact pairs of the FE model to model the lung sliding and investigate the disease-induced change of lung sliding motion. As shown in Fig. 6, the effect of different friction conditions on lung deformation could be simulated through adjusting friction coefficients. If a more serious lung disease occurs, such as pleural invasion by peripheral lung cancer or chest wall invasion (Sakuma et al. 2017), local lung sliding motion can be completely restricted, often requiring surgical correction. In such a case, the cohesive interaction behaviour can be defined for the contact pair between a tumour and the chest wall to model the attachment of the tumor with the chest wall in the FE model.

The results presented in Section 3 show that the accuracy of FE simulations is affected by model parameters, such as Poisson's ratio, friction coefficient and tissue heterogeneity, but a simple biomechanical model with a homogeneous frictionless model can provide a fairly good registration results, an average TRE of $3.8 \mathrm{~mm}$, as shown in Table 2, which is ensured by the success criteria of FE simulations, deforming an FE model to $99.5 \%$ of its target volume. In general, it is difficult to accurately measure or estimate these parameters. However, the effects of their uncertainties on image registration accuracy can be reduced in the proposed method by adding an image registration step to compensate for displacement residuals of an initial biomechanical simulation using estimated model parameters. With the proposed method, the changes of these model parameters did not 
show a significant impact on the registration accuracy in terms of final TRE. Therefore, introducing this step makes it possible to reduce the complexity and the computational time of the biomechanical modelling in the first step by employing a simple homogeneous biomechanical model without compromising the overall registration performance. To further speed up the algorithm, the parallel implementation of finite element methods on GPU (Han et al. 2014b) could be adopted. The registration accuracy of the hybrid method can also be improved. Following the method by Zhong et al (Zhong et al., 2012), the estimated displacement fields from the hybrid method may be used to improve the registration accuracy in low-contrast regions, where one expects that the deformation estimation is less accurate due to homogeneous image intensity. More specifically, we can remesh and re-run the FE model by only applying the displacements estimated from the hybrid method to those nodes of the FE model lying outside of low-contrast region as displacement boundary conditions, and recalculate the displacement distributions of these low-contrast regions. Thus, the execution time and registration accuracy of the hybrid method could be further improved in the future.

\subsection{Potential applications and ongoing work}

The hybrid method has provided a good registration accuracy comparable to some state-of-the-art intensity-based image registrations, meanwhile introducing biomechanical models facilitates various physical and physiological properties modelling of the lungs, such as sliding motion, heterogeneity of tissue stiffness, friction, pressure difference etc., within one single FE model, such an integrated solution is unknown for intensity-based image registration methods. In the proposed method, biomechanical modelling in the first step estimates most of physically realistic deformations of the lungs, and only a relatively small deformation residual need to be recovered with intensity-based image registration in the second step, thus reducing the chance of creating physically unrealistic deformation. This advantage of the hybrid method over intensity-based image registration methods needs to be further investigated and confirmed with phantom tests (Kim et al. 2016) in which a ground truth can be generated.

Since the displacement compensation in the intensity-based image registration process reflects the distributions of the prediction errors of biomechanical modelling, it has a potential to be used for analysing the factors affecting the accuracy of biomechanical 
modelling. For example, as indicated in Section 3 , based on the analysis of displacement compensation patterns, the simulation accuracy of biomechanical models can be improved by introducing a small amount of friction for Case 1 or considering the tissue heterogeneity for Case 8.

In the framework of the proposed method, we can also estimate model parameters, such as friction coefficient, Young's modulus and Poisson's ratio, with an optimisation process through perturbation within realistic reported ranges determined from in vivo/in vitro experiments or experience values (Han et al., 2012, Amelon, 2012; Li et al., 2013), and minimising the required displacement compensation from intensity-based image registration could be an ideal objective function. The estimations of model parameters can potentially be used for the diagnosis and assessment of lung diseases. For example, pleural effusion, a condition in which excess fluid accumulated within the pleural space, and pleural adhesion and pleural invasion by peripheral lung cancer, all of these diseases can cause the change of lubrication within the pleural cavity locally or globally. The assessment on the friction/sliding condition of the lung surfaces can provide aids for physicians in deciding whether a tumour has invaded into the chest wall, and whether extensive surgery is necessary in the treatment planning (Sakuma et al., 2017). This potential application needs to be explored.

Due to the predictive capacity of biomechanical models, the developed method has a potential to be used in adaptive radiotherapy. For example, accurate margins for tumour motion are very important for accurate tumour targeting and sparing healthy tissues from radiation. However, tumours and healthy tissues may change in shape, location and stiffness during the course of treatment, which may affect the deformation and motion of tumours and the lungs. Moreover, patient's breathing pattern changes from time to time. Therefore, the estimated margin in the treatment planning, based on non-rigid intensitybased image registration on $4 \mathrm{D} \mathrm{CT}$ data, may not represent the real margin for the delivery; there is a risk of missing the target or causing unnecessary radiation exposure on normal tissues. Although the motion models based on non-rigid intensity-based image registration, incorporating with surrogates, are capable of predicting the lung motion over a complete normal breathing cycle, its prediction capacity on the motion and deformation of tumours and inner lung tissues is limited, when subjected to breathing irregularity 
and the changes of tumours and healthy tissues. However, physics and physiology based biomechanical models of the lungs can address this limitation. Through assessing the impacts of these changes on motion and deformation of the tumours and the lungs, a new modified FE model incorporating these changes can be constructed and used to generate an FE-estimated motion model. Then, the FE-estimated motion model is refined with the image registration process of the hybrid method using the FE-estimated CT image and a treatment $\mathrm{CT}$ image (e.g. cone-beam $\mathrm{CT}$ ), to provide a revised motion model and tumour trajectory, thus helping radiation oncologists to adjust the radiation treatment plan adaptively in order to prevent insufficient radiation dose to the tumours and excessive radiation dose to the healthy tissues during the course of treatment.

In future work, we plan to investigate extending our method to incorporate information on displacement compensation from image registration into an optimisation scheme for model parameters extraction (e.g. heterogeneous tissue distribution/tissue mechanical properties, friction, non-uniform pleural pressure distribution (Fuerst et al., 2015), boundary constraints et al), with the aim of determining a more accurate physically realistic biomechanical motion model of the lung and the distribution of stiffness of lung tissues and pressure distribution which may be directly related with the respiratory function of lungs (Li et al. 2013, Fuerst et al., 2015).

\section{Conclusion}

In this paper, we have proposed a hybrid biomechanical-model based image registration method for lung motion estimation in which sliding motion could be explicitly modelled. The proposed method consists of two consecutive processes: patient-specific biomechanical modelling followed by intensity-based image registration. Patient-specific biomechanical modelling simulates biomechanical behaviour of tissues and captures physically plausible deformation, while image-registration process is used for displacement compensation to biomechanical modelling by making full use of intensity patterns of medical images. The proposed method has been evaluated on lung motion estimation. A quantitative comparison to three representative registration approaches for lung motion estimation shows that the hybrid method could provide good registration accuracy when recovering lung deformation, especially in the near-surface regions, which is particularly 
relevant to radiotherapy applications involving the treatment of mobile, superficial tumours. The preliminary study on the effect of parameters in biomechanical models to deformation fields has found that model parameters (Poisson's ratio, friction) and the tissue heterogeneity affect the accuracy of biomechanical modelling in the first process of the proposed registration method, although they have no obvious impact on final registration performance of the proposed method. It has also demonstrated that the proposed method has the potential in optimising patient-specific biomechanical models through analysing the pattern of displacement compensation from the image-registration process, if the purpose of applications is to develop more accurate, predictable, physical models.

\section{Acknowledgements}

The authors would like to acknowledge the financial support from EPSRC program Grant EP/F025750/1 and EP/H046410. The work is also sponsored by Shanghai Pujiang Programme(16PJ1409400) and the Thousand Talents Programme (China). The constructive comments of the anonymous reviewers have improved the paper.

\section{References}

Al-Mayah, A., Moseley, J., Brock, K.K., 2008. Contact surface and material nonlinearity modeling of human lungs. Physics in Medicine and Biology 53, 305-317.

Al-Mayah, A., Moseley, J., Velec, M., Brock, K.K., 2009. Sliding characteristic and material compressibility of human lung: Parametric study and verification. Medical Physics 36, 4625-4633.

Al-Mayah, A., Moseley, J., Velec, M., Hunter, S., Brock, K., 2010. Deformable image registration of heterogeneous human lung incorporating the bronchial tree. Medical Physics 37, 4560-4571.

Al-Mayah, A., Moseley, J., Velec, M., Hunter, S., Brock, K., 2011. Toward efficient biomechanical-based deformable image registration of lungs for image-guided radiotherapy. Physic in Medicine and Biology $56,4701-4513$

Amelon, R., 2012. Development and charactrization of a finite element model of lung motion. Ph.D. thesis. University of Iowa.

Amelon, R., Cao, K., Christensen, G., Raghavan, M.A., 2014. A measure for characterizing sliding on lung boundaries. 2014;42(3):642-650. Annals of Biomedical Engineering 42, 642-650.

Castillo, E., Castillo, R., Martinez, J., Shenoy, M., Guerrero, T., 2010. Four-dimensional deformable image registration using trajectory modeling. Physics in Medicine and Biology 55, 305-327.

Castillo, R., Castillo, E., Guerra, R., Johnson, V.E., McPhail, T., Garg, A.K., Guerrero, T., 2009. A framework for evaluation of deformable image registration spatial accuracy using large landmark point sets. Physics in Medicine and Biology 54, 1849-1870.

Delmon, V., Rit, S., Pinho, R., Sarrut, D., 2013. Registration of sliding objects using direction dependent B-splines decomposition. Physics in Medicine and Biology 58, 1303-1314.

Fuerst, B., Mansi, T., Carnis, F., M, S., xe, lzle, Zhang, J., Declerck, J., Boettger, T., Bayouth, J., Navab, N., Kamen, A., 2015. Patient-specific biomechanical model for the prediction of lung motion from 4-D CT images. IEEE Transactions on Medical Imaging 34, 599-607.

Galetke, W., Feier, C., Muth, T., Ruehle, K.H., Borsch-Galetke, E., Randerath, W., 2007. Reference values for dynamic and static pulmonary compliance in men. Respiratory Medicine 101, 1783-1789. 
Gray, H., 1918. Anatomy of the Human Body. Lea\&Febiger, Philadelphia.

Han, L., Hawkes, D., Barratt, D., 2014a. A hybrid biomechanical model-based image registration method for sliding objects, in: SPIE Medical Imaging, pp. G1-G6.

Han, L., Hipwell, J.H., Eiben, B., Barratt, D., Modat, M., Ourselin, S., Hawkes, D.J., 2014b. A nonlinear biomechanical model based registration method for aligning prone and supine MR breast images. IEEE Transactions on Medical Imaging 33, 682-694.

Han, L., Hipwell, J.H., Tanner, C., Taylor, Z., Mertzanidou, T., Cardoso, J., Ourselin, S., Hawkes, D.J., 2012. Development of patient-specific biomechanical models for predicting large breast deformation. Physics in Medicine and Biology 57, $455-472$.

Heinrich, H.P., Jenkinson, M., Brady, M., Schnabel, J.A., 2013. Mrf-based deformable registration and ventilation estimation of lung CT. IEEE Transactions on Medical Imaging 32, 1239-1248.

Heinrich, M., Jenkinson, M., Brady, M., Schnabel, J., 2010. Discontinuity preserving regularisation for variational optical-flow registration uisng the modified Lp norm, in: Medical Image Analysis for the Clinic-A Grand Challenge, Workshop MICCAI 2010.

Hipwell, J.H., Vavourakis, V., Han, L., Mertzanidou, T., Eiben, B., Hawkes, D.J., 2016. A review of biomechanically informed breast image registration. Physics in Medicine and Biology 61, R1.

Holden, M., 2008. A review of geometric transformations for nonrigid body registration. IEEE Transactions on Medical Imaging 27, 111-128.

Kim, J., Saitou, K., Matuszak, M.M., Balter, J.M., 2016. A finite element head and neck model as a supportive tool for deformable image registration. International Journal of Computer Assisted Radiology and Surgery 11, 1311-1317.

Klein, S., Staring, M., Murphy, K., Viergever, M.A., Pluim, J.P.W., 2010. Elastix: A toolbox for intensity-based medical image registration. IEEE Transactions on Medical Imaging 29, $196-205$.

Li, M., Castillo, E., Zheng, X.L., Luo, H.Y., Castillo, R., Wu, Y., Guerrero, T., 2013. Modeling lung deformation: A combined deformable image registration method with spatially varying Young's modulus estimates. Medical Physics Letter 40, 1-10.

Li, P., Malsch, U., Bendl, R., 2008. Combination of intensity-based image registration with 3D simulation in radiation therapy. Physics in Medicine and Biology 53, 4621-4637.

Maintz, J.B.A., Viergever, M.A., 1998. A survey of medical image registration. Medical Image Analysis $2,1-36$.

McClelland, J.R., Blackall, J.M., Tarte, S., Chandler, A.C., Hughes, S., Ahmad, S., Landau, D.B., Hawkes, D.J., 2006. A continuous 4D motion model from multiple respiratory cycles for use in lung radiotherapy. Medical Physics 33, 3348-3358.

McClelland, J.R., Hawkes, D.J., Schaeffter, T., King, A.P., 2013. Respiratory motion models: A review. Medical Image Analysis 17, 19-42.

Muers, M.F., 2003. Lung cancer. Medicine 31, 28-37.

Murphy, K., Ginneken, B.v., Reinhardt, J.M., Kabus, S., Ding, K., Deng, X., Cao, K., Du, K., Christensen, G.E., Garcia, V., Vercauteren, T., Ayache, N., Commowick, O., Malandain, G., Glocker, B., Paragios, N., Navab, N., Gorbunova, V., Sporring, J., Bruijne, M.d., Han, X., Heinrich, M.P., Schnabel, J.A., Jenkinson, M., Lorenz, C., Modat, M., McClelland, J.R., Ourselin, S., Muenzing, S.E.A., Viergever, M.A., Nigris, D.D., Collins, D.L., Arbel, T., Peroni, M., Li, R., Sharp, G.C., SchmidtRichberg, A., Ehrhardt, J., Werner, R., Smeets, D., Loeckx, D., Song, G., Tustison, N., Avants, B., Gee, J.C., Staring, M., Klein, S., Stoel, B.C., Urschler, M., Werlberger, M., Vandemeulebroucke, J., Rit, S., Sarrut, D., Pluim, J.P.W., 2011. Evaluation of registration methods on thoracic CT: The EMPIRE10 challenge. IEEE Transactions on Medical Imaging 30, 1901-1920.

Pace, D.F., Enquobahrie, A., Hua, Y., Aylward, S.R., Niethammer, M., 2011. Deformable image registration of sliding organs using anisotropic diffusive regularization, in: Biomedical Imaging: From Nano to Macro, 2011 IEEE International Symposium on, pp. 407-413.

Permutt, S., Bromberger-Barnea, B., Bane, H., 1962. Alveolar pressure, pulmonary venous pressure, and the vascular waterfall. Medical Thoraces 19, 239-260.

Rietzel, E., Chen, G.T.Y., 2006. Deformable registration of 4D computed tomography data. Medical Physics 33, 4423-4430.

Risser, L., Vialard, F.X., Baluwala, H.Y., Schnabel, J.A., 2013. Piecewise-diffeomorphic image registration: Application to the motion estimation between 3D CT lung images with sliding conditions. Medical Image Analysis 17, 182-193.

Ruan, D., Esedoglu, S., Fessler, J.A., 2009. Discriminative sliding preserving regularization in medical image registration, in: Biomedical Imaging: From Nano to Macro, 2009. ISBI '09. IEEE International Symposium on, pp. 430-433.

Rueckert, D., Sonoda, L.I., Hayes, C., Hill, D.L.G., Leach, M.O., Hawkes, D.J., 1999. Nonrigid registra- 
tion using free-form deformations: application to breast MR images. IEEE Transactions on Medical Imaging 18, 712-721.

Sakuma, K., Yamashiro, T., Moriya, H., Murayama, S., Ito, H., 2017. Parietal pleural invasion/adhesion of subpleural lung cancer: Quantitative 4-dimensional CT analysis using dynamic-ventilatory scanning. European Journal of Radiology 87, 36-44.

Samavati, N., Velec, M., Brock, K., 2015. A hybrid biomechanical intensity based deformable image registration of lung 4D CT. Physics in Medicine and Biology 60, 3359-3373.

Schmidt-Richberg, A., Ehrhardt, J., Werner, R., Handels, H., 2012a. Fast explicit diffusion for registration with direction-dependent regularization, in: Dawant, B., Christensen, G., Fitzpatrick, J.M. Rueckert, D. (Eds.), Biomedical Image Registration. Springer Berlin Heidelberg. volume 7359 of Lecture Notes in Computer Science. book section 23, pp. 220-228.

Schmidt-Richberg, A., Werner, R., Handels, H., Ehrhardt, J., 2012b. Estimation of slipping organ motion by registration with direction-dependent regularization. Medical Image Analysis 16, 150-159.

Sotiras, A., Davatzikos, C., Paragios, N., 2013. Deformable medical image registration: A survey. IEEE Transactions on Medical Imaging 32, 1153-1190.

Staring, M., Klein, S., Pluim, J.P.W., 2007. Nonrigid registration with tissue-dependent filtering of the deformation field. Physics in Medicine and Biology 52, 6879-6892.

Vandemeulebroucke, J., Bernard, O., Rit, S., Kybic, J., Clarysse, P., Sarrut, D., 2012. Automated segmentation of a motion mask to preserve sliding motion in deformable registration of thoracic CT. Medical Physics 39, 1006-1015.

Villard, P., Beuve, M., Shariat, B., Baudet, V., Jaillet, F., 2005. Simulation of lung behaviour with finite elements: influence of bio-mechanical parameters, in: Third International Conference on Medical Information Visualisation - Biomedical Visualisation, pp. 9-14.

Werner, R., Ehrhardt, J., Schmidt, R., Handels, H., 2009a. Patient-specific finite element modeling of respiratory lung motion using 4D CT image data. Medical Physics 36, 1500-1511.

Werner, R., Ehrhardt, J., Schmidt-Richberg, A., Handels, H., 2009b. Validation and comparison of a biophysical modeling approach and non-linear registration for estimation of lung motion fields in thoracic 4D CT data, in: SPIE Medical Imaging, pp. 72590U-72590U.

West, J.B., Dollery, C.T., Naimark, A., 1964. Distribution of blood flow in isolated lung; relation to vascular and alveolar pressures. Journal of Applied Physiology 19, 713-724.

Wu, Z., Rietzel, E., Boldea, V., Sarrut, D., Sharp, G.C., 2008. Evaluation of deformable registration of patient lung 4D CT with subanatomical region segmentations. Medical Physics 35, 775-781.

Yin, Y., Hoffman, E., Lin, C.L., 2010. Lung lobar slippage assessed with the aid of image registration, in: Jiang, T., Navab, N., Pluim, J.W., Viergever, M. (Eds.), Medical Image Computing and ComputerAssisted Intervention MICCAI 2010. Springer Berlin Heidelberg. volume 6362 of Lecture Notes in Computer Science. book section 71, pp. 578-585.

Yushkevich, P., Piven, J., Cody, H., Ho, S., Gee, J.C., Gerig, G., 2005. User-guided level set segmentation of anatomical structures with ITK-SNAP

Zhang, T., Orton, N.P., Mackie, T.R., Paliwal, B.R., 2004. Technical note: A novel boundary condition using contact elements for finite element based deformable image registration. Medical Physics 31, $2412-2415$.

Zhong, H., Kim, J., Li, H., Nurushev, T., Movsas, B., Chetty, I.J., 2012. A finite element method to correct deformable image registration errors in low-contrast regions. Physics in Medicine and Biology $57,3499-3515$. 